\title{
TREATMENT OF VULVAR HAILEY-HAILEY DISEASE WITH LOW-DOSE NALTREXONE
}

\author{
Marina Sousa Gomes ${ }^{1}$, João Pedro Prata1, Vera Teixeira², Paula Pinheiro ${ }^{1}$ \\ ${ }^{1}$ Department of Obstetrics and Gynecology, Alto Minho Local Healthcare Unit, Viana do Castelo, Portugal, Viana do \\ Castelo, Viana do Castelo, Portugal; ${ }^{2}$ Department of Dermatology, Alto Minho Local Healthcare Unit, Viana do Castelo, \\ Portugal, Viana do Castelo, Viana do Castelo, Portugal
}

Problem statement: Familial benign pemphigus, or Hailey-Hailey disease (HHD), is a rare and severe autosomal dominant dermatosis characterized by chronic recurrent vesicles, erosions, and maceration in intertriginous areas. Despite the multiple therapies, such as topical and systemic corticosteroids, systemic immunomodulators, topical and systemic retinoids and laser, HHD can be significantly difficult to control. In nongeneralized HHD, there are some case reports of HHD treated successfully with low-dose naltrexone.

We report a case of vulvar Hailey-Hailey disease treated with low-dose naltrexone.

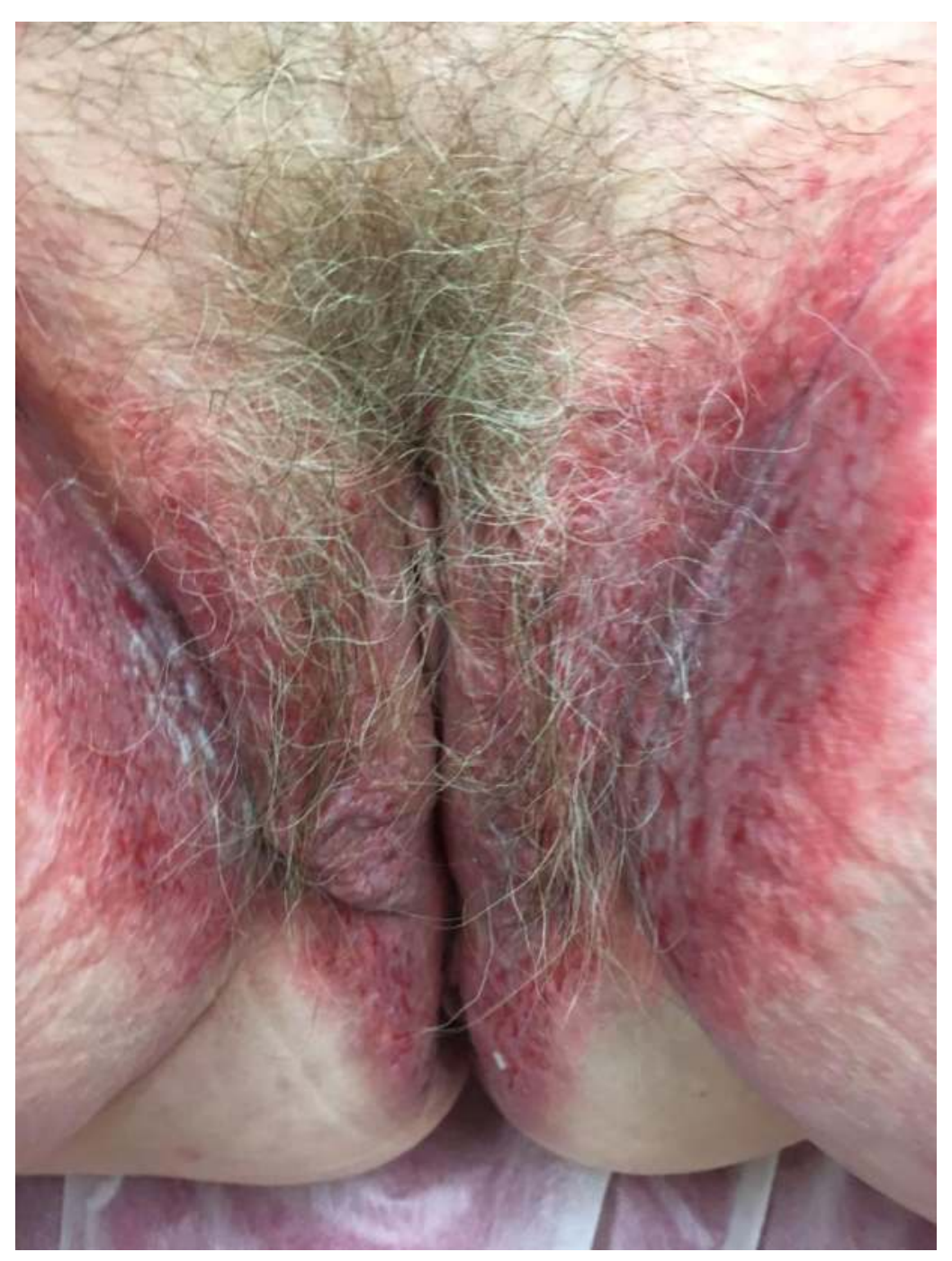

Figure 1: Before naltrexone treatment
Results: A 71-year-old white woman presented with multiple large eroded plaques on the vulva (figure 1). She had attempted topical corticosteroids and topical and oral antifungal agents, with no improvement in the symptoms and size of the lesions. She had a 10-year history of painful erosions. She had no clinically family history of HHD. Vulvar biopsy has proven HHD. Treatment with low-dose naltrexone, $3 \mathrm{mg}$ nightly (with titration to $4.5 \mathrm{mg}$ ), and minocycline $100 \mathrm{mg}$ daily was initiated. She maintained topical corticosteroids and antifungal agents. The lesions began to heal and five months later her skin was with no lesions (figure 2). There was an improvement in pruritus and pain. She didn't note adverse effects of low-dose naltrexone.

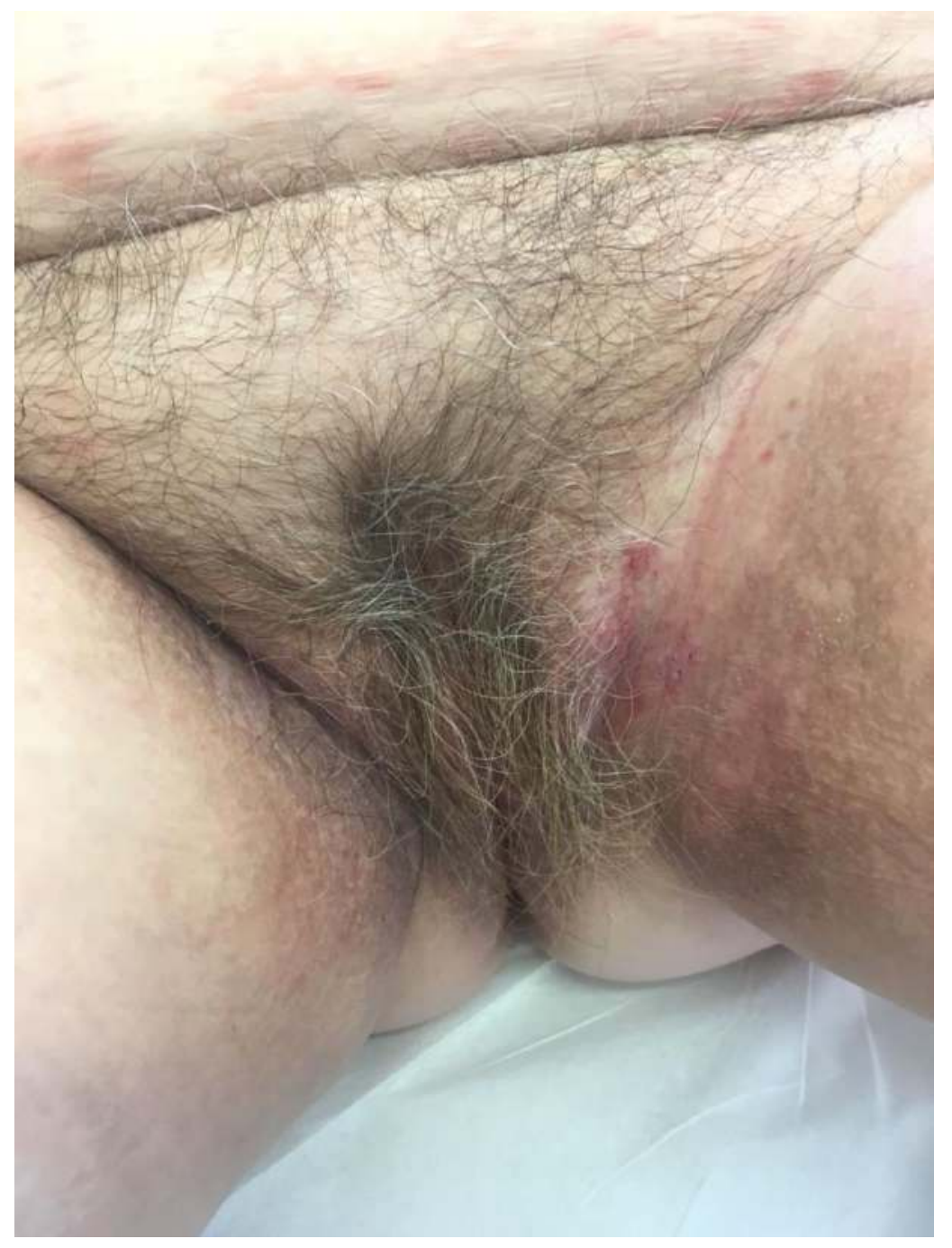

Figure 2: After naltrexone treatment

Conclusion: Low-dose naltrexone may represent an effective and successful treatment modality in HHD without serious adverse effects. Low-dose naltrexone can influence opioid or toll-like receptor signaling and improve calcium mobilization and keratinocyte differentiation. These mechanisms can be responsible for wound healing. More studies are needed to clarify the mechanism and to define the role of low-dose naltrexone for the treatment of HHD. This successful case suggests low-dose naltrexone can be considered a novel therapy for HHD. 